\title{
Expression of Interleukins (ILs) mRNA in Otitis Media
}

\author{
Sung Ho Kang ${ }^{1}$, Dae Joon Lim ${ }^{1}$, Bo Hyung Kim ${ }^{1}$, Myung Sang Yu ${ }^{1}$, Shinseok Kang ${ }^{2}$, \\ ${ }^{1}$ Department of Otorhinolaryngology of Medicine School, Kunkuk University, Chungju, Republic of Korea \\ ${ }^{2}$ Joongbu branch, Chungbuk Veterinary Service Laboratory, Chungju, Republic of Korea
}

\section{Email address:}

kkdin@kku.ac.kr (S. Ho Kang), lim516@kku.ac.kr (D. J. Lim), bhkim@kku.ac.kr (Bo H. Kim), dryums@gmail.com (M. S. Yu), newstonek@naver.com (S. Kang)

${ }^{*}$ Corresponding author

\section{To cite this article:}

Sung Ho Kang, Dae Joon Lim, Bo Hyung Kim, Myung Sang Yu, Shinseok Kang. Expression of Interleukins (ILs) mRNA in Otitis Media. Biochemistry and Molecular Biology. Vol. 4, No. 4, 2019, pp. 59-66. doi: 10.11648/j.bmb.20190404.12

Received: October 3, 2019; Accepted: October 21, 2019; Published: October 25, 2019

\begin{abstract}
The purpose of this study was to investigate the expression of interleukins (ILs) cytokine in postauricular skin, mastoid mucosa, inflamed mastoid mucosa and middle ear mucosa from Otitis patients. Specimens were obtained from patients with otitis media who visited the Department of Otorhinolaryngology, Chungju Hospital, Kunkuk University School of Medicine, Chungju, Republic of Korea between August 2015 and July 2017. We examined human 38 kinds of human interleukins in this experiment. The total RNA was extracted from specimens using RNAiso Plus (Takara, Japan) according to the manufacturer's instructions. 38 ILs primers were used. IL-4, IL-8, IL-9, IL-21, IL-24 were not expressed at postauricular skin, mastoid mucosa, inflamed mastoid mucosa, and middle ear mucosa. IL-1a, IL-17, IL-17d, IL-17e and IL-17f were strongly expressed in mastoid mucosa and middle ear mucosa. The degree of IL-7 gene expression may be an indicator gene in otitis media. IL-1a, IL-17, IL-17d, IL-17e and IL-17f were involved in the inflammatory and immune processes of otitis media.
\end{abstract}

Keywords: Otitis Media, Interleukins (ILs), Inflamed Mastoid Mucosa, Middle Ear Mucosa

\section{Introduction}

Otitis media is a disease that causes inflammation in middle ear. It is divided into rapid development and chronic development like other infectious diseases. The acute type occurs within a short period accompanying severe pain. The chronic type does not cause pain and lasts for a long time. Most cases are when the exudates come out.

A common cause of otitis media is dysfunction of the Eustachian tube due to inflammation of mucous membranes in nasopharynx. The major causative organisms are Haemophilus influenzae, Moraxella catarrhalis, and Streptococcus pneumoniae [1-3].

Interleukins (ILs) are a class of cytokines and refers to immunomodulatory proteins that elicit various responses in cells and tissues. These cytokines contain a large number of known immunological 'second messenger' molecules in mammals. It is known that interleukin binds to the watersoluble receptor on the cell surface and initiates the reaction [4]. The majority of interleukin cytokines can be divided into molecule families based on structure and function.

Interleukins (ILs) divided into 8 groups that is the IL-1 family, the common $\gamma$ chain cytokine family, the IL-10 family, the IL-12 family, the type 2 immune response cytokines family, IL-17 family, the chemokine activity interleukin family, the other interleukins. The IL-1 family consists of IL1, IL-18, IL-33, IL-36, IL-37 and IL-38. The common $\gamma$ chain cytokine family consists of IL-2, IL-4, IL-7, IL-9, IL-15 and IL-21. IL-10, IL-19, IL-20, IL-22, IL-24, IL-26, IL-28A, IL$28 \mathrm{~B}$ and IL-29 belongs to the IL-10 family. IL-12, IL-23, IL27 and IL-35 are a member of the IL-12 family. The members of the type 2 immune response cytokine family are IL-5, IL-13, IL-25 (IL-17e) and IL-31. IL-17a, IL-17b, IL$17 \mathrm{c}$, IL-17d and IL-17f belong to the IL-17 family. The chemokine activity interleukin family is IL-8 and IL-16. The other interleukins consists of IL-3, IL-6, IL-11, IL-14, IL-32 and IL-34 [5]. The IL-1 family is derived from macrophages, B cells, monocytes, dendritic cells, Th1-cells macrophages, and target cells are $\mathrm{T}$ helper cells, B cells, NK cells, macrophages, and endothelium, which are known to be involved in proinflammatory and anti-inflammatory 
responses [6, 7]. The common $\gamma$ chain cytokine family is made up from $\mathrm{T}$ cells and dendritic cells, NKT cells, eosinophils, mast cells, stromal cells, epithelial cells, fibroblasts, monocytes, and stimulate the CD4 + T cells, CD4, CD8 T cells, NK cells, B cells. transmition signals from one cell to another and direct programs of cellular differentiation are their main function [5]. The source of the IL-10 family of cytokines are monocytes, Th2 cells, CD8 T cells, mast cells, macrophages and stimulates macrophages, mast cells, B cells, Th1 cells, Th2 cells. IL-10 family cytokines were emerged before the adaptive immune response. It was reported that the IL-10 family cytokines were essential for maintaining the integrity and homeostasis of tissue epithelial layers. Members of this family can promote innate immune responses from tissue epithelia to limit the damage caused by viral and bacterial infections. These cytokines can also facilitate the tissue-healing process in injuries caused by infection or inflammation. The IL-10 family cytokines have indispensable functions in many infectious and inflammatory diseases [8]. The signaling of IL 12 family is mediated by members of the Janus kinase-signal transducers and activators of transcription (JAK-STAT) family. IL 12 family members are involved in driving T-helper cell development and are related to granulomatous inflammatory diseases [9]. The type 2 immune responses cytokines family promotes antihelminth immunity, neutralizes toxins, maintains metabolic homeostasis, and regulates wound repair and tissue regeneration pathways following infection or injury. Nevertheless, when type 2 responses are dysregulated, they can become important drivers of disease. Type 2 immunity induces a complex inflammatory response characterized by eosinophils, mast cells, basophils, type 2 innate lymphoid cells, IL-4-and/or IL-13-conditioned macrophages and T helper 2 (TH2) cells, which are crucial to the pathogenesis of many allergic and fibrotic disorders. As chronic type 2 immune responses promote disease, the mechanisms that regulate their maintenance are thought to function as crucial disease modifiers [10]. The chemokine activity interleukins family is produced from macrophages, lymphocytes, epithelial cells, endothelial cells, eosinophils, and CD8 + T cells, and is known to have functions of neutrophil chemotaxis and CD4 + chemoattractant [5]. The interleukins17 family is have functions as a pro-inflammatory factor secreted by activated T-cells. The IL 17 family functions in kinds of tissues, such as brain, articular cartilage, lung, meniscus, intestine, hematopoietic tissue, bone, kidney and skin. IL 17 family members are essential participants in various arms of the adaptive immune response and are the key to understand cytokine networks which coordinate innate and adaptive immunity to certain pathogens $[11,12]$. Since the occurrence of otitis media is often caused by infection, we started this study to investigate the gene expression of interleukins due to the immune activity, which are caused by inflammation.

\section{Materials and Methods}

\subsection{Materials}

\subsubsection{Specimens}

Specimens were obtained from patients with otitis media who visited the Department of Otorhinolaryngology, Chungju Hospital, Kunkuk University School of Medicine, Chungju, Republic of Korea between August 2015 and July 2017. Ethical approval was obtained from the Institutional Ethics Committee of Kunkuk University School of Medicine (KUCH 2015-08-037). All subjects provided written informed consent. The specimens, such as postauricular skin, mastoid mucosa, inflamed mastoid mucosa and middle ear mucosa were collected and stored at $-70^{\circ} \mathrm{C}$ until used.

\subsubsection{Primers for Interleukins (ILs)}

In this study, 38 ILs primers (Table 1) were used. The primer sequence of ILs used GenBank's information.

Table 1. Primers sequences of Interleukins (ILs) mRNA for semi-quantitative RT-PCR.

\begin{tabular}{|c|c|c|c|c|}
\hline Gene & Forward & Reverse & Product (bp) & Reference \\
\hline IL-1a & CCTGGGCATTCTTGTTTCAATT & CAGAGAATTTTGTTGCAAGCTTTA & 240 & NM_000575 \\
\hline IL-1b & CGGCCACATTTGGTTCTAA & AGAGCACACCAGTCCAAATTG & 203 & NM_000576 \\
\hline IL-3 & TTATTGCCTGGAGTGTGAACTGTA & ACATCCCTAGGAACCAGTTTAAAA & 132 & NM_000588 \\
\hline IL-4 & ACAAGCAGCTGATCCGATTCC & TCGTCTTTAGCCTTTCCAAGAAGT & 129 & M13982 \\
\hline IL-5 & CCAAGAAAGAGTCAGGCCTTAATT & CCTGGAGGAAAATACTTCAATGAT & 147 & NM_000879 \\
\hline IL-6 & GATTGTTGTTGTTAATGGGCATTC & GGCTCTGAAACAAAGGATATTCAA & 276 & M54894 \\
\hline IL-7 & GTCTTCTTCTGTGCTGGAGATGT & ACCATTTATTGAAGGCACTGTGT & 180 & J04156 \\
\hline IL-8 & AGCTGGACATTAAAGAGGAAAGAG & TTCCAGAAGTTTCTTTTGTTCTAT & 263 & M28130 \\
\hline IL-9 & TGGGCATTCCCTCTGACAA & GGCTGTTCACAGGAAAAATATGG & 166 & NM_000590 \\
\hline IL-10 & AATTGCTTGAACCCAGGAGAT & ATGTAACATTCCCAGAGGAATTGA & 173 & NM_000572 \\
\hline IL-11 & GGTCCCGGATTCTTGGGT & GTCCCCACCCCAACATGAA & 127 & M57765 \\
\hline IL-12a & ATTGCTAAGAAGGGAAAATATCCA & TAGCTCAGATGCTTTCATGATTA & 175 & NM_000882 \\
\hline IL-12B & GATGGATGGGAACGCAAGAGATA & GTGGCCCTAAATTGCTTTATCAAC & 140 & NM_002187 \\
\hline IL-13 & GTTTGTCACCGTTGGGGATTG & TGACCCTGCCTCGGATGAG & 185 & NM_002188 \\
\hline IL-14 & CCAGGCACCTGCTCCTCTT & AGTCCCTGGCTGTTGTGAATACC & 156 & NM_175852 \\
\hline IL-15 & AGTCCGGAGATGCAAGTATTCA & TGCCGAGTGTTTTGTTATGTCTAA & 267 & U14407 \\
\hline
\end{tabular}




\begin{tabular}{lllll}
\hline Gene & Forward & Reverse & Product (bp) & Reference \\
\hline IL-16 & AAAGGAGCAGCCTCAGAACAAA & GAGGCTTTTCTCCTGATGACAA & 162 & AF053412 \\
IL-17 & CGGAATTGTCTCCACAACACCT & AAGGAAAATGGGAAGGGCAGAA & 282 & AY630567 \\
IL-17a & ATACAAGAAGTTCTGGGAGGAAC & GTGGTGCCTTGATCAGACAG & 171 & NM_002190 \\
IL-17b & CACGGTGCCTGTGTCTG & ACAGCGATGGTCTCCATGA & 166 & AF152098 \\
IL-17c & CCCGTGTCTGGAGGACAGC & CAGGACAGGGAGCCAAGGTA & 144 & NM_-13278 \\
IL-17d & GAAAGCCACTGGGGCCAAT & TGCTAACGTGGCCCACTTTG & 158 & NM_138284 \\
IL-17e & CCAGCACAGGCACTTTCTAGAT & CAATAACAGCTTCCTCCTCAGAAT & 164 & AF305200 \\
IL-17f & CGTTCCCATCCAGCAAGAGAC & TGGGTAAGGAGTGGCATTTCTACA & 186 & NM_052872 \\
IL-18 & GGAAATGAATCCTCCTGATAACA & GTCTTCGTTTTGAACAGTGAACAT & 220 & AY044641 \\
IL-19 & GCTTGATGACAAGGAACCTGTAT & AGGCCCTTTCAGCAGATAGAGT & 215 & AY040367 \\
IL-20 & GGATGGGTTGTGGATAAGTTTTG & ATGCTGGCCGTGTAGGATT & 127 & NM_018724 \\
IL-21 & AGTCCTGGCAACATGGAGAG & CAGGAAAAAGCTGACCACTCAC & 224 & AF254069 \\
IL-22 & TCTTGGCCCTCTTGGTACA & TTCTCCCCAATGAGACGAAC & 166 & NM_020525 \\
IL-24 & GGTTTACCCTGCTTCTCTGGA & CCGGGCACTCGTGATGTTAT & 162 & BT007156 \\
IL-28c & CCATATCCTCTCCCAGCTCCG & TTCAGGTCTCGCGTGAGGAG & 180 & AY336717 \\
IL-29 & ACCTCAAATATGTGGCCGATG & AACTCAGCCCTATGTCTCAGTCAG & 171 & AY129150 \\
IL-31 & GGAGGGCTGAGATGTGAATTTG & CCAGGGAGCATTGACAACTCTTA & 190 & NM_001014336 \\
IL-32 & ATCGCGGAGGTGGGTTTC & AGCCATGTGGAACTGTGAGTCA & 136 & NM_001308078 \\
IL-33 & TTACTTTTGCTTTGGAGGATGAA & GGCAGTGGTTTTCACACTTATGG & 238 & AY905581 \\
IL-34 & GGTGCTGCCCTCACTGTCC & CAGGCTGGGGGGTCATAGA & 132 & BC029804 \\
IL-36a & CTTCAGGACCAGACGCTCATA & GGCTGGTCCCCGACTTTA & 176 & NM_014440 \\
\hline
\end{tabular}

\subsection{Methods}

\subsubsection{Total RNA Extraction}

To examine the expression of interleukins genes, the total RNA was extracted from specimens using RNAiso Plus (Takara, Japan) according to the manufacturer's instructions. Briefly, approximately $20 \mathrm{mg}$ of tissue was taken and $1 \mathrm{~mL}$ of RNAiso Plus reagent was added to the tissue, and then tissue was completely disrupted using a homogenizer. After adding $200 \mathrm{ul}$ of chloroform (Sigma, USA), the mixture was vigorously mixed and centrifuged at 13,000 rpm for 10 minutes using a refrigerated centrifuge (Labogene, Korea). $500 \mathrm{ul}$ of the supernatant was removed into a new tube, and the same amount of isopropanol (Sigma, USA) was added, and then mixed gently. The tube was kept at room temperature for 10 minutes, and then centrifuged at 13,000 rpm for 10 minutes. After the supernatant was discarded, the precipitated pellet was rinsed gently with $1 \mathrm{~mL}$ of $70 \%$ ethanol and centrifuged again at 13,000rpm for 10 minutes. After discarding the supernatant, the pellet was dried at room temperature for 10 minutes and then, dissolved with 30 ul of DEPC-treated RNase- and DNase free water (WEL GENE, Korea). The extracted RNA was kept at $-70^{\circ} \mathrm{C}$ until used.

\subsection{2. $R T$ - PCR}

Complementary DNA (cDNA) was synthesized using PrimeScript $1 \mathrm{st}$ strand cDNA synthesis kit (Takara Clontech, Japan) according to manufacturer's recommendations. In brief, the first mixture containing $1 \mathrm{ul}$ of Oligo dT Primer, 1 ul of dNTP mixture, 1 ul of extracted RNA, 7 ul of DEPC-treated RNase- and DNase-free water was incubated at $65^{\circ} \mathrm{C}$ for 5 minutes and immediately chilled on ice. Then, the second mixture containing $4 \mathrm{ul}$ of $5 \times$ - PrimeScript buffer, 0.5 ul of RNase inhibitor, 1 ul of PrimeScript RTase, 4.5 ul of DEPC-treated water were incubated at $50^{\circ} \mathrm{C}$ for 1 hour and at $95^{\circ} \mathrm{C}$ for 5 minutes. PCR primers were designed to target interleukins genes using the Primer 3 software (http://frodo.wi.mit.edu/) using the genomic information obtained from NCBI site (Table 1). As an internal control, primers for $\beta$-actin gene were used.

PCR reaction for amplification was performed using $2 \times$ TOPsimple DyeMix-multi HOT (Enzynomics, Korea). PCR reaction were carried out using SimpleAmp PCR machine (ABI, USA) with the PCR mixture composing of 2 ul of cDNA, $0.5 \mu \mathrm{L}$ of each primer, $7 \mathrm{ul}$ of DEPC-treated water, and 10 ul of $2 \times$-TOPsimple DyeMix-multi HOT (Enzynomix, Korea) in a total volume of $20 \mathrm{ul}$. The thermal cycling conditions were 10 minutes at $95^{\circ} \mathrm{C}$, followed by 35 cycles of 30 seconds at $95^{\circ} \mathrm{C}, 30$ seconds at $60^{\circ} \mathrm{C}, 1$ minute at $72^{\circ} \mathrm{C}$, and final extension for 5 minutes at $72^{\circ} \mathrm{C}$ for and ILs. The PCR products were separated by electrophoresis on $1.5 \%$ agarose gel at $100 \mathrm{~V}$ for 1 hour, and the gels were stained with ethidium bromide and photographed using a Gel Doc system (Bio-Rad Laboratories, Hercules, CA, USA). All experiments were performed in triplicate.

\section{Results}

\subsection{Subjects}

The 27 patients having otitis media were grouped as postauricular skin $(n=9)$, mastoid mucosa $(n=7)$, inflamed mastoid mucosa $(n=4)$ and middle ear mucosa $(n=7)$, and consisted of 15 males and 12 females, ranging in age from 19 71 years. 


\subsection{Interleukins (ILs) Genes mRNA Expression in Otitis Media Specimens}

The expression of interleukins gene in otitis media is shown in Figure 1.

Among the interleukin genes, IL-4, IL-8, IL-9, IL-21 and IL-24 showed no immune response related to otitis media. In the postauricular skin, the IL-17 family showed a weak response, whereas no other ILs mRNA was expressed. The IL-1a was significantly expressed. In mastoid mucosa, IL-15 and IL-33 were not expressed, but IL-1a, IL-17, IL-16, IL17f, IL-32, IL-36a, IL-34, IL-6, IL-5, IL-10, IL-1b and IL-2 were significantly expressed. IL-13, IL-7, IL-11, IL-12, IL13, IL-14, IL-17a, IL-17b, IL-17c, IL-18, IL-19, IL-20 were expressed but no significant difference was observed to affect immunity.

Inflamed mastoid mucosa, All of IL-1a, IL-1b, IL-2, IL-3, IL-4, IL-5, IL-6, IL-7, IL-8, IL-9, IL-10, IL-11, IL-12a, IL12b, IL-13, IL-14, IL-15, IL-16, IL-17, IL-17a, IL-17b, IL17c, IL-17d, IL-17e, IL-17f, IL-18, IL-19, IL-20, IL-21, IL-
22, IL-24, IL-28c, IL-29, IL-31, IL-32, IL-33, IL-34, IL-36a were all expressed, IL-1a, IL-6, IL-7, IL-10, IL-12a, IL-16, IL-17, IL-17d, IL-17e, IL-17f, IL-18, IL-19, IL-20, IL-22, IL- 28c, IL-32, IL-36a were strongly expressed among these interleukins. In middle ear mucosa, IL-4, IL-8, IL-9, IL-21, and IL-24 were not expressed and IL-15 and IL-33 expression levels were very low. The expression level of the remaining ILs was similar to that of inflamed mastoid mucosa. The results of this experiment showed that the major interleukins involved in immunization in otitis media were IL-1a, IL-7, IL-10, IL-16, IL-17d, IL-17e, IL-17f, IL-18, IL19, IL-22, IL-28c, IL-32 and IL-36a. The genes of IL-4, IL-8, IL-9, IL-21, IL-24 were not expressed or partly be expressed in otitis media. In particular, IL-7 and IL-9 are known to be highly similar in function [11], but they seem to play a different role in otitis media immunity. The results of this study show that various factors are involved in otitis media immune activity. In particular, the role of interleukins is expected to be further studied.
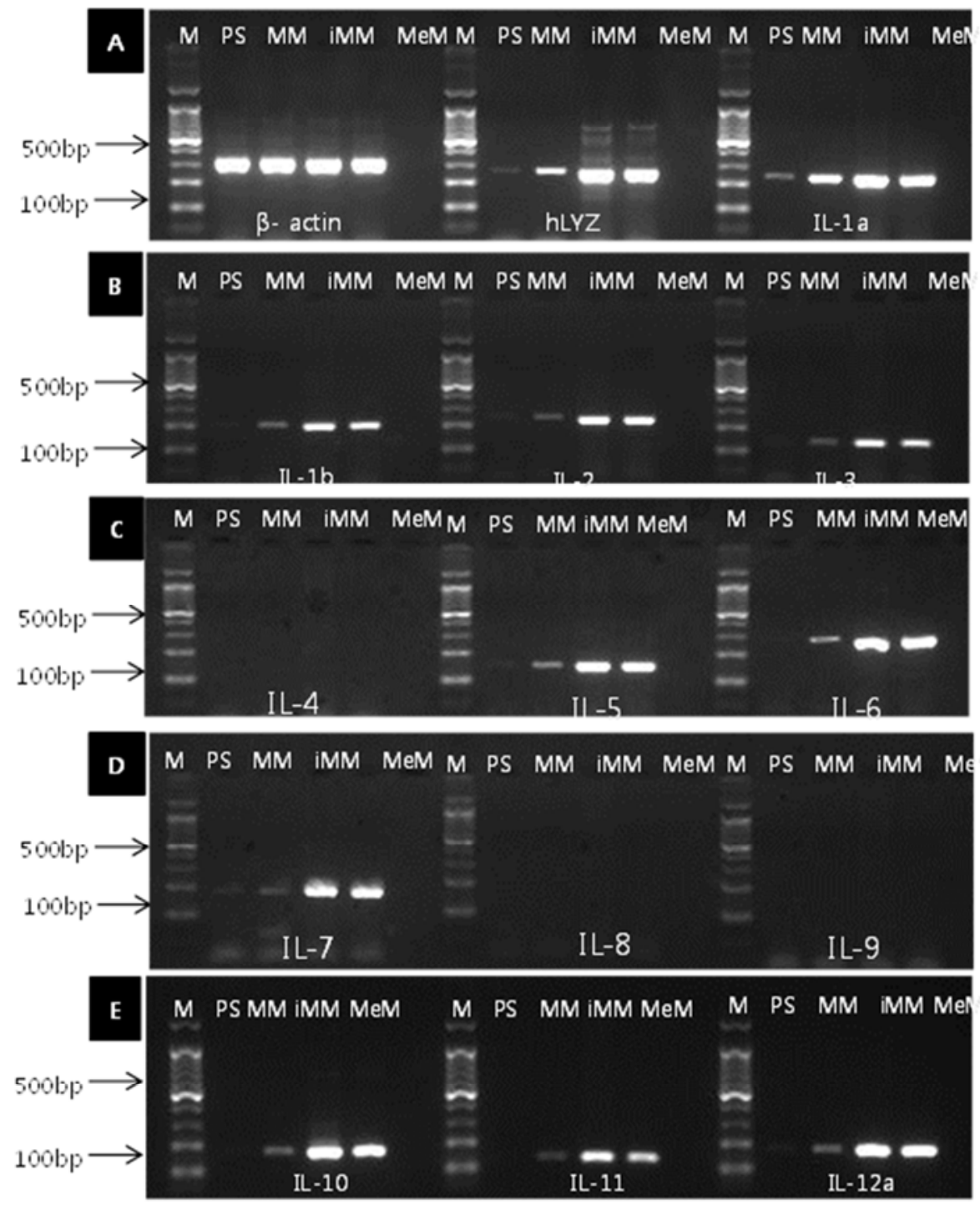

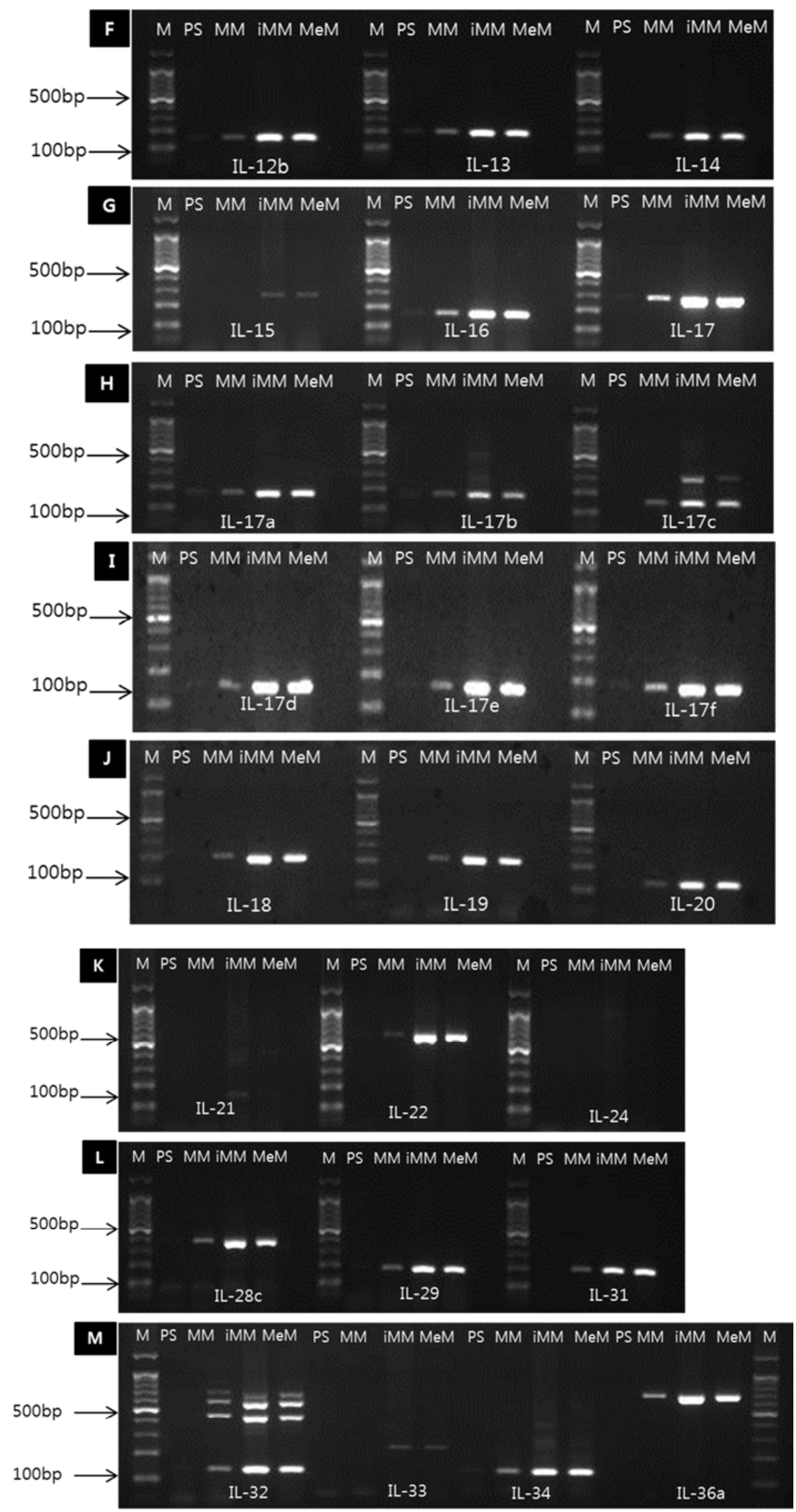

Figure 1. Expression of Interleukin genes in the middle ear tissue was analyzed by RT-PCR. PCR products were separated by $1.5 \%$ agarose gel electrophoresis, stained with ethidium bromide (E-Q). Results are representative of three experiments. M: 100bp DNA ladder, PS: Postauricular skin, MM: Mastoid mucosa, iMM: Inflamed mastoid mucosa, MeM: Middle ear mucosa; IL: Interleukin. 


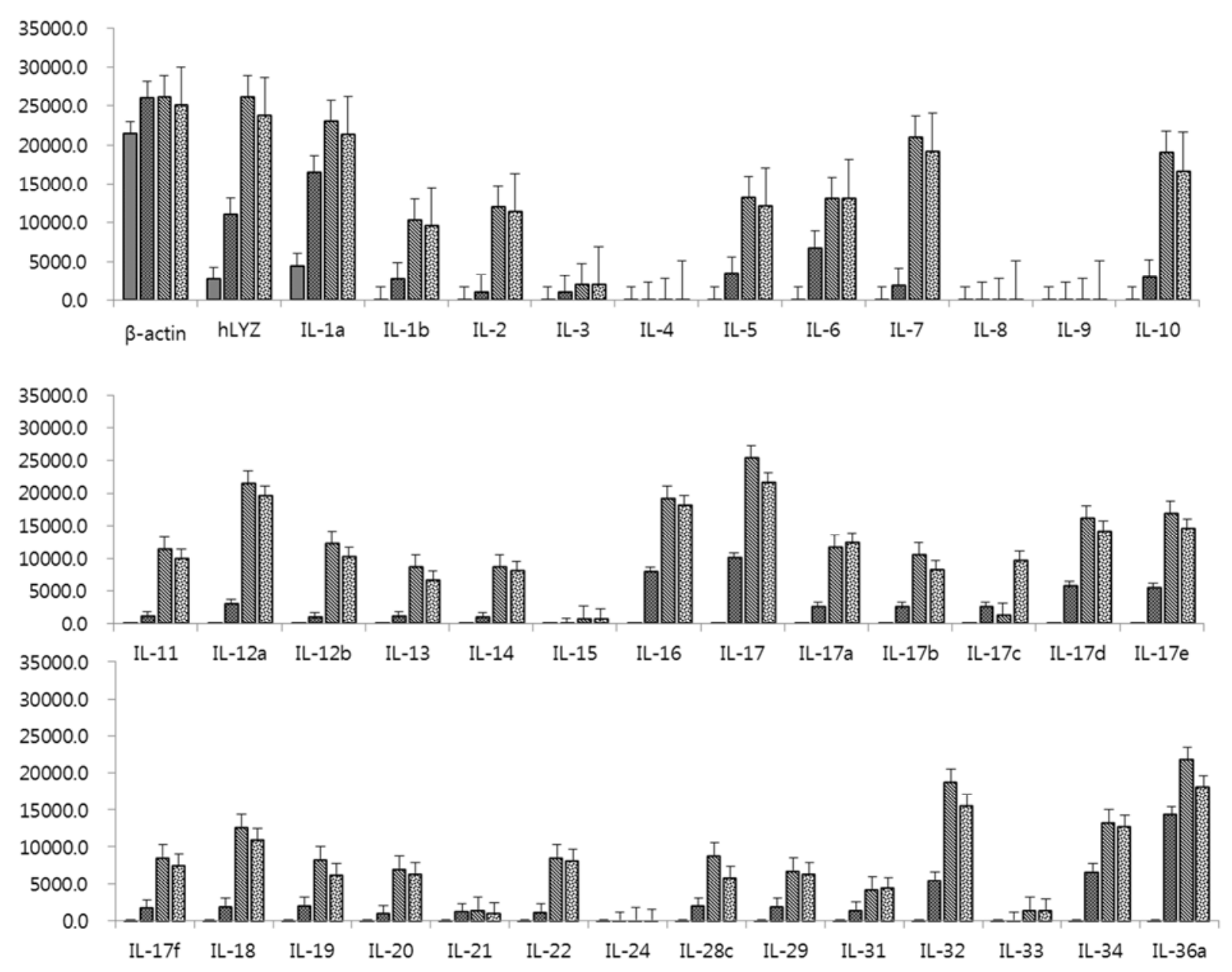

Figure 2. Relative mRNA Expression levels of Interleukin genes in the middle ear tissue was analyzed by ImageJ.; IL: Interleukin,

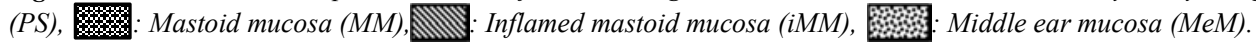

\section{Discussion}

Postauricular skin is located behind the auricle of the ear. Postauricular cutaneous mastoid fistula due to otitis media is very rare because it acts as a defense against direct infection from the outside. The mastoid is the portion of the petrous temporal bone that lies superior to the middle ear cavity. The mastoid is filled with a system of interconnecting air-filled cells. The mastoid antrum serves as an open canal between the middle ear and mastoid air cells. Adjust the temperature freely and smooth the gas exchange in the mastoid cell area is thought to be a main function of the mastoid mucosa. Mastoid mucosa plays an important role in the formation and maintenance of mastoid air cells. The loss of mastoid air cells due to damage to the mastoid mucosa is presumed one of reasons why antibiotics treatments are not effective due to exudates in otitis media. If foreign matter such as a pathogen enters the middle ear, it may cause contraction and occlusion of blood vessels around the infection area, which may ultimately lead to a decrease in middle ear pressure. The microchannel and vasculars are connected to the mastoid mucosa. These results indicate that the mastoid mucosa is sensitive to the vasoactive mediators that can play a role in middle ear pressure regulation and play an important role in the formation and elimination of otitis media [13, 14].

Middle ear mucosa is one of the defense systems that protects the middle ear from the pathogen in the middle ear. The presence of mucociliary defense system in middle ear is seen as the first line of defense. Secretion by the mucosa has a profound biological significance. Immunoglobulins $A, G$, and even $\mathrm{E}$ and $\mathrm{M}$ are produced locally by the mucosa and may contribute to the immunodefense of the middle ear. Secretory lysozyme is also produced by the mucosa and may contribute enzymatic defense of the ear. Mucosal immunoglobulins and lysozyme are significantly elevated in the effusions, which would imply that local defense systems are hyperactive in OME. It also appears that these increases are related to the increase of the secretory cell population. The middle ear also can transport macromolecules very rapidly across intact mucosal epithelium [15].

It was reported that cytokines are small secreted proteins released by cells have a specific effect on the interactions and communications between cells. Cytokine is a general name; other names include lymphokine (cytokines made by lymphocytes), monokine (cytokines made by monocytes), chemokine (cytokines with chemotactic activities), and interleukin (cytokines made by one leukocyte and acting on 
other leukocytes). Cytokines may act on the cells that secrete them (autocrine action), on nearby cells (paracrine action), or in some instances on distant cells (endocrine action). There are both pro-inflammatory cytokines and anti-inflammatory cytokines [16].

In this study, IL-4, IL-8, IL-9, IL-21 and IL-24 were not involved in otitis media immune responses at all. IL-1a, IL-5, IL-6, IL-7, IL-10, IL-12, IL-16, IL-17, IL-17d, IL-17e, IL17f, IL-22, IL-32, IL-34 and IL-36a appear to be strongly involved in inflammation and immune responses in inflamed mastoid mucosa and middle ear mucosa in otitis media. In the case of IL-1a, IL-17, IL-17d, IL-17e and IL-17f mRNA expression level was equal or stronger than that of lysozyme.

The IL-1 family, the IL-10 family, the IL-12 family, the type 2 immune response cytokines family, the chemokine activity interleukins family, the IL-17 family, the other interleukins were found in the otitis media. These results indicate that the interleukin response in otitis media does not manifest itself in a interleukin family form because of the source cells of interleukin and target cells of interleukins are differ each.

The IL-17 family is most strongly involved in otitis media when viewed as a family unit.

IL-1a and IL-36 genes were expressed in the IL-1 family. These are thought to stimulate $\mathrm{T}$ helper cells and increase $\mathrm{T}$ cell responses and dentritic cells. IL-7 was expressed only in the common $\gamma$ chain cytokine family. IL-7 is a cytokine that increases the proinflammatory response and may be used as an otitis media recovery gene. The IL- 17 family is known to play a central role in the control of inflammation during bacterial infections. In this experiment, the IL-17 family was strongly expressed when inflammation progressed [17]. In the IL-10 family, IL-10 and IL-22 are expressed and stimulate macrophages and Th1 cells, leading to increased cytokines secretion and wound healing tissue. IL-12 was expressed only in the IL-12 family, suggesting that increase the IFN- $\gamma$ and TNF- $\alpha$ secretion from NK cells stimulation. IL-5 and IL-17e were expressed in the type 2 immune response cytokines family, IL-5 presumed that stimulates Th2 cells to promote $\mathrm{B}$ cell differentiation and IgA production. IL-17e increase the induction of IgE, IgG. IL-16 was expressed in the chemokine activity interleukins family and induces CD4 + chemoattractant. IL-17b, IL-17d and IL-17f were expressed in the IL-17 family, but IL-17d and IL-17f were strongly expressed among the contents of the IL-17 family. These are thought to induce antimicrobial peptides, proinflammatory cytokines and chemokines. Other expressed IL-6 stimulates $\mathrm{T}$ cells and $\mathrm{B}$ cells to increase the differentiation and vitality of T-cells and B-cells, and seems to involve the production of $\operatorname{IgG}, \operatorname{IgM}$, and IgA. IL-32 and IL-34 stimulate macrophages and monocytes to induce TNFa, IL-8 and IL-6 secretion. It is presumed that the microglial proliferation of the inflammatory site is increased.

\section{Conclusion}

1) IL-1a, IL-6, IL-7, IL-10, IL-12a, IL-16, IL-17, IL-17d, IL-17e, IL-17f, IL-18, IL-19, IL-20, IL-22, IL- 28c, IL-
32, IL-36a were strongly expressed in an inflamed mastoid mucosa.

2) IL-1a, IL-17, IL-16, IL-17f, IL-32, IL-36a, IL-34, IL-6, IL-5, IL-10, IL-1b and IL-2 were significantly expressed but IL-15 and IL-33 were not expressed in mastoid mucosa.

3) The expression levels of IL-15, IL-33 were very low and IL-4, IL-8, IL-9, IL-21, IL-24 were not expressed in middle ear mucosa.

4) The IL-17 family showed a weak response but no other ILs mRNA was expressed in the postauricular skin.

5) IL-1a, IL-17, IL-17d, IL-17e and IL-17f were strongly expressed in mastoid mucosa and middle ear mucosa of the patients and appears to be the main interleukins involved in inflammatory progression and immune response in otitis media.

6) The IL-17 family mainly involved in otitis media. The severity of inflammation may be determined by the degree of IL-7 gene expression.

\section{Acknowledgements}

This study was supported by research fund from Dong-A ST Co., Ltd (Seoul, Republic of Korea).

\section{Conflict of Interest}

The authors have no conflicts of interest to disclose.

\section{References}

[1] Minovi A, Dazert S. Diseases of the middle ear in childhood Review. GMS Current Topics in Otorhinolaryngology - Head and Neck Surgery. 2014; 13: 1-29.

[2] Coker TR, Chan LS, Newberry SJ, et al. Diagnosis, microbial epidemiology, and antibiotic treatment of acute otitis media in children: a systematic review. JAMA. 2010; 304 (19): 21612169 .

[3] Casey JR, Pichichero ME. Changes in frequency and pathogens causing acute otitis media in 1995-2003. Pediatr Infect Dis J. 2004; 23 (9): 824-828.

[4] Commins SP, Borish L, Steinke JW. Immunologic messenger molecules: Cytokines, interferons, and chemokines. J Allergy Clin. Immunol. 2010; 125: 53-72.

[5] Akdis M, Aab A, Altunbulakli C, Azkur K, Costa RA. Crameri R, Duan S, Eiwegger T, Eljaszewicz A, Ferstl R, Frei R, Garbani M, Globinska A, Hess L, Huitema C, Kubo T, Komlosi Z, Konieczna P, Kovacs N, Kucuksezer UC, Meyer N, Morita H, Olzhausen J, Mahony LO, Pezer M, Prati M, Rebane A, Rhyner C, Rinaldi A, Sokolowska Milena, Stanic B, Sugita K, Treis A, Veen W, Wanke K, Wawrzyniak M, Wawrzyniak P, Wirz OF. Zakzuk JS, Akdis CA. Interleukins (from IL-1 to IL-38), interferons, transforming growth factor $\mathrm{b}$, and TNF-a: Receptors, functions, and roles in diseases. Fundamentals of allergy and immunology. 2016; 984-1011.

[6] Dinarello CA. Interleukin-1 in the pathogenesis and treatment of inflammatory diseases. Blood. 2011; 117 (14): 3720-3732. 
[7] Veerdonka FL, Stoeckmanc AK, Wu G, Boeckermann AN, Azama T, Netea MG, Joosten LA, Meer WM, Hao R, Kalabokis V, Dinarelloa CA. IL-38 binds to the IL-36 receptor and has biological effects on immune cells similar to IL-36 receptor antagonist. PNAS, 2012; 109 (8): 3001-3005.

[8] Ouyang W, Rutz S, Crellin NK, Valdez PA, Hymowitz SG. Regulation and functions of the IL-10 family of cytokines in inflammation and disease. Annu Rev Immunol 2011; 29: 71109.

[9] Vignali DA, Kuchroo VK. IL-12 family cytokines: immunological playmakers. Nat Immunol. 2012; 13 (8): 722728.

[10] Thomas AW. Type 2 cytokines: mechanisms and therapeutic strategies. Nature Reviews Immunology. 2015; 15: 271-282.

[11] Weaver CT, Hatton RD, Mangan P, Harrington LE. IL-17 Family Cytokines and the expanding diversity of effector $\mathrm{T}$ Cell lineages. Annu. Rev. Immunol. 2007; 25: 821-852.
[12] Moseley TA, Haudenschild DR, Rose L, Reddi AH. Interleukin-17 family and IL-17 receptors. Cytokine \& growth factor reviews, 2003; 14 (2): 155-174.

[13] Holmquist J, Jarlstedt J, Tjellström A. Surgery of the mastoid in ears with middle ear effusion. Ann Otol Rhinol Laryngol Suppl. 1980; 89 (3): 322-323.

[14] Fooken Jensen PV, Gaihede M. Congestion of mastoid mucosa and influence on middle ear pressure - Effect of retroauricular injection of adrenaline. Hear Res. 2016; 340: 121-126.

[15] Lim DJ. Functional morphology of the mucosa of the middle ear and Eustachian tube. Ann Otol Rhinol Laryngol. 1976; 85 (2): 36-43.

[16] Zhang JM, AnJianxiong. Cytokines, Inflammation and Pain. Int Anesthesiol Clin. 2007; 45 (2): 27-37.

[17] Monin L, Gaffen S L, IL-17 family cytokines: signaling mechanisms, biological activities and therapeutic implications. Cold Spring Harb Perspect Biol. 2018; 10 (4). 